\title{
ESTUDIO PILOTO DEL TRATAMIENTO COADYUVANTE DE LA CISTITIS RECIDIVANTE DE LA MUJER MEDIANTE AM3 (INMUNOFERON囚)
}

\author{
F. MILLÁN RODRÍGUEZ, F. ROUSAUD BARÓN, A. ROUSAUD BARÓN
}

Servicio de Urología. Fundación Puigvert. Barcelona.

Actas Urol Esp. 27 (3): 196-201, 2003

\section{RESUMEN}

"ESTUDIO PILOTO DEL TRATAMIENTO COADYUVANTE DE LA CISTITIS RECIDIVANTE DE LA MUJER MEDIANTE AM3 (INMUNOFERON®)"

OBJETIVOS: Evaluar la eficacia del AM3 (Inmunoferon ${ }^{\circledR}$ ) como coadyuvante del tratamiento antibiótico en la cistitis recidivante de la mujer (CRM) para conocer la proporción de respuesta al mismo con la intención de diseñar posteriormente un ensayo clínico.

MATERIAL Y MÉTODOS: Se estudiaron consecutivamente 24 mujeres que presentaron en los seis últimos meses dos episodios documentados de cistitis no asociada a otra patología y que no respondieron al tratamiento antibiótico. Se les pautó tratamiento antibiótico y 3 g diarios de AM3 durante 9 meses. Se evaluó la presencia de infección y de sintomatología miccional irritativa en la visita inicial, y al primer, tercer, sexto y noveno mes.

RESULTADOS: Completaron el estudio 19 pacientes. La proporción de infección disminuyó del 100\% en la visita inicial al $26 \%$ al primer mes para estabilizarse en torno al $50 \%$ en los meses sucesivos. La sintomatología miccional irritativa disminuyó del $46 \%$ en la visita inicial a proporciones inferiores al 10\% en los meses sucesivos.

CONCLUSIÓN: El AM3 redujo la presencia de infección urinaria manifiesta en un 50\% y la sintomatología miccional irritativa característica de CRM en un 90\%. Estos hechos sugieren la necesidad de realizar estudios controlados para confirmar el efecto de AM3 en esta patología.

PALABRAS CLAVE: Cistitis recidivante. AM3. Inmunoferon®.

\section{ABSTRACT \\ "MODEL STUDY OF THE TREATMENT OF RECURRENT CYSTITIS IN WOMEN BY MEANS OF AM3 (INMUNOFERON®)"}

OBJECTIVES: The aim is to evaluate the effectiveness of AM3 (Inmunoferon $®$ ) in the treatment of the recurrent cystitis in women in order to know the rate of good results, previously to design a clinical trial.

MATERIAL AND METHODS: Twenty-four women who had been diagnosed of two cystitis episodes in the previous 6 months without cure by antibiotic treatment were admitted to the study. Standard antibiotic treatment and 3 daily grammes of AM3 was given for 9 months. Infection and irritative symptoms during micturition rate were evaluated at the inclusion date and afterwards, at the first, third, sixth and nineth month.

RESULTS: Nineteen patients finished the study. The infection rate decreased from $100 \%$ at the inclusion date to $26 \%$ in the first month and then it became stable about $50 \%$. Irritative symptoms during micturition decreased from $46 \%$ at the inclusion date to a rate lower than $10 \%$ in the 4 controls running.

CONCLUSION: AM3 reduced evident urinary infection in a 50\% and irritative symptoms during micturition in a $90 \%$. Control clinical trials are needed to confirm the AM3 effects on this pathology.

KEY WORDS: Recurrent cystitis. AM3. Inmunoferon® 
$\mathrm{M}$ ás de un $60 \%$ de mujeres tienen alguna infección del tracto urinario en su vida ${ }^{1}$ y en un 10 $20 \%$ de casos se trata de cistitis recidivantes ${ }^{2}$. La cistitis recidivante de la mujer (CRM) se define como 3 o más episodios de infección urinaria no complicada en una mujer documentada por cultivo urinario en los últimos 12 meses sin que existan anomalías estructurales o funcionales. Los posibles mecanismos etiopatogénicos son por un lado, todos aquellos factores que interfieren en los mecanismos de defensa y por otro aquellos que facilitan la entrada de uropatógenos. Entre los que interfieren los mecanismos de defensa están la disminución de la IgA secretora (por lesiones epiteliales o inmunosupresión), o la disminución de la excreción de glicoproteína 1 (por bacterias o estados inflamatorios). En cuanto a los posibles factores que facilitan la entrada de uropatógenos, están la actividad sexual (por el masaje uretral), el ser portadoras de un dispositivo intrauterino (por la angulación uretral), la constipación intestinal que provoca una superpoblación de $E$. Coli o la diarrea crónica que provoca una alteración del equilibrio bacteriano. El diagnóstico diferencial ha de realizarse con la cistitis intersticial y con el síndrome uretral.

El tratamiento clásico de la CRM es la quimioprofilaxis con dosis baja de antibiótico ya sea diaria o post-coital durante períodos largos de tiem$\mathrm{po}^{3-6}$. El problema es que tras suspenderse la quimioprofilaxis, aparece una reinfección en el 60\% de los casos en 3 meses $^{7}$. Es por ello, que se han ensayado tratamientos coadyuvantes mediante inmunoestimulantes o biológicos (extractos bacterianos), vacunas ${ }^{8}$, glicosaminoglicanos, tratamientos hormonales, y hasta zumo de arándanos. El tratamiento hormonal más usado es la administración sistémica o tópica de estrógenos, que provocan una disminución del $\mathrm{pH}$ vaginal, cambios en los receptores celulares, una disminución del estímulo inflamatorio y un aumento de la síntesis de glicoproteína 1. Entre los tratamientos coadyuvantes experimentados, los más recientes son la aplicación tópica vaginal de Lactobacilli ${ }^{9-10}$. Se han publicado trabajos que han evaluado la acción de la ingesta de zumo de arándanos agrios (Vaccinium oxycocos) que contiene proantocianidinas que interfieren en el desarrollo de las fimbrias de la E. Coli y por tanto merma su capacidad de adherencia a las células uroteliales ${ }^{11-15}$.
El AM3 (Inmunoferon ${ }^{\circledR}$ ) es una asociación no covalente de un polisacárido (glucomanano $\alpha 1$ $6,1,2$ ) de peso molecular $15010^{3}$ obtenido de la Candida utilis y una proteína formada por un heterodímero rico en glutamina de peso molecular 11 $10^{3}$ obtenida de semillas no germinadas de Ricinus communis. El 98,2\% restante es una matriz inorgánica de $\left(\mathrm{CaSO}_{4} 2 \mathrm{H}_{2} \mathrm{O}\right)_{2}$ y $\left(\mathrm{CaHPO}_{4} 2 \mathrm{H}_{2} \mathrm{O}\right)_{5}$. Diferentes estudios in vitro e in vivo han demostrado su acción sobre el sistema inmunitario, al actuar sobre dos niveles ${ }^{16-35}$. Primero, potencia la resistencia del huésped frente a diversos patógenos extra e intracelulares mediante su acción sobre la inmunidad de mediación celular. Por otro lado, no sólo no interfiere con las acciones de antibióticos ni antivirales sino que potencia su acción. Es por ello, que puede tener un gran interés realizar un ensayo clínico para evaluar la eficacia del AM3 como coadyuvante del tratamiento antibiótico en la CRM. No obstante, para diseñar dicho ensayo clínico previamente ha de evaluarse si dicho tratamiento puede conseguir posibles efectos beneficiosos y conocerse la proporción de respuesta al mismo. Para ello, se ha diseñado este estudio piloto cuyo objetivo es valorar la proporción de pacientes afectas de CRM que responden positivamente al tratamiento de AM3 como coadyuvante del tratamiento antibiótico, con la intención de si la respuesta es satisfactoria poder disenar posteriormente un ensayo clínico que refrende los resultados obtenidos en este estudio piloto.

\section{MATERIAL Y MÉTODOS}

Para evaluar la proporción de respuesta al tratamiento se estimó una muestra de 20 pacientes, a la que se añadió un $20 \%$ en previsión de posibles missing, por lo que al final la muestra fue de 24 pacientes. Las pacientes se reclutaron de forma consecutiva a partir del 15 de abril de 2001 siguiendo los criterios de inclusión y exclusión. Se incluyó cualquier mujer que hubiera presentado en los 6 últimos meses 2 episodios documentados de infección urinaria baja sintomática o asintomática que no hubiera respondido al tratamiento antibiótico y a las medidas higiénicas. Se excluyeron aquellos casos en los que la infección pudiera atribuirse a otra patología como litiasis infecciosa o patología obstructiva o aquellos casos de infección urinaria febril o de vías urinarias altas. En la 
visita de inclusión se recogió si las pacientes presentaban sintomatología miccional irritativa, se les aconsejó medidas higiénicas (ingesta líquida superior a 2 litros diarios, micciones frecuentes y lavado y micción post-coital), se les pautó tratamiento antibiótico según antibiograma y finalmente se les suministró $3 \mathrm{~g}$ diarios de AM3 durante 9 meses. En ningún caso se pautó tratamiento antibiótico supresivo ni profilaxis post-coital. En los casos de infección vaginal concomitante demostrada se pautó también tratamiento antibiótico en óvulos vaginales. A continuación se realizaron controles clínicos y con sedimento urinario el primer, el tercer, el sexto y el noveno mes. Asimismo se registró la tolerancia a la medicación pautada y la posible presencia de efectos adversos. Las variables dependientes del estudio fueron dos: la presencia de sintomatología miccional irritativa y la presencia de infección urinaria, ambas medidas como proporción en las respectivas visitas de control.

\section{RESULTADOS}

El estudio fue completado por 19 pacientes a los 9 meses habiéndose perdido 5. Concretamente se produjo una pérdida en el control del primer mes, otra en el tercer mes, dos en el sexto mes y una en el noveno mes. Todos los casos fueron abandonos voluntarios no relacionados con intolerancia a la medicación.

Todas las pacientes presentaban infección urinaria en el momento de la inclusión en la visita inicial, siendo el germen más frecuente la $E$. Coli. De las 24 pacientes, $10(41,7 \%)$ habían tenido 2 episodios demostrados de infección urinaria y $14(58,3 \%)$ más de 2 episodios. Asimismo, 11 pacientes $(45,8 \%)$ presentaban sintomatología miccional irritativa y las otras 13 $(54,2 \%)$ se encontraban asintomáticas. De las 24 pacientes, $7(29,2 \%)$ tenían infección vaginal concomitante. Siguiendo el antibiograma las pacientes fueron tratadas en el $75 \%$ de los casos con ciprofloxacina, en el $12,5 \%$ con amoxicilina/clavulánico, el 8,3\% con cotrimoxazol y el $4,2 \%$ con fosfomicina.

En el control del primer mes sólo una paciente refirió epigastralgia y otra sensación de mareo, ambos cuadros de curso autolimitado y que no provocaron el abandono del tratamiento con AM3.
El resto de las pacientes $(91,6 \%)$ presentó una buena tolerancia. La proporción de pacientes con infección urinaria se redujo a un 26,1\% (Fig. 1) y sólo un 8,7\% presentaban sintomatología miccional irritativa (Fig. 2). Todos los casos de infección urinaria fueron por E. Coli . Como tratamiento se pautó ciprofloxacina en el $45,5 \%$ de los casos, amoxicilina/clavulánico y fosfomicina en el $27,3 \%$.

En el control del tercer mes sólo un 4,5\% de las pacientes presentaban sintomatología miccional irritativa (Fig. 2) y un $45,5 \%$ de las pacientes tenían infección urinaria (Fig. 1). De éstas, en el 90\% de los casos, la E. Coli era el germen causante. Un $50 \%$ de las pacientes se trató con ciprofloxacina, un $25 \%$ con amoxicilina/clavulánico y el otro $25 \%$ con cotrimoxazol.

En el control del sexto mes un 10\% de las pacientes presentaban sindrome miccional irrita-

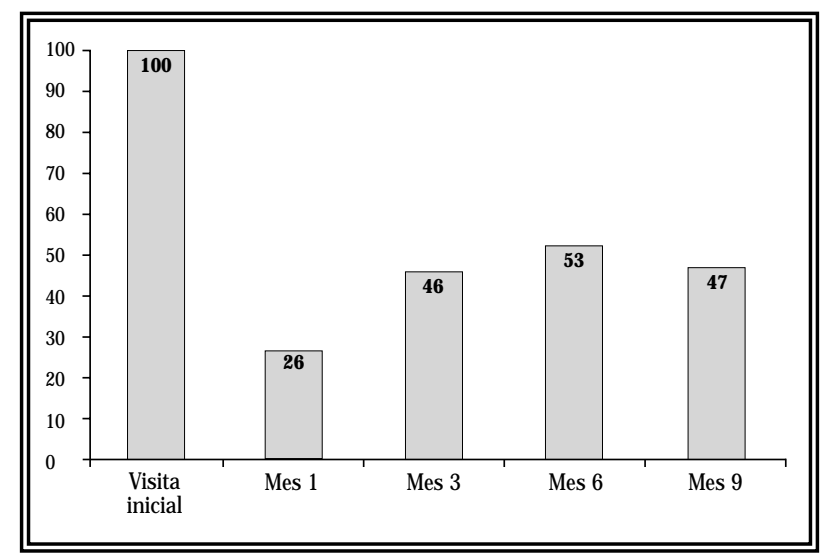

FIGURA 1. Proporción de infección urinaria (\%).

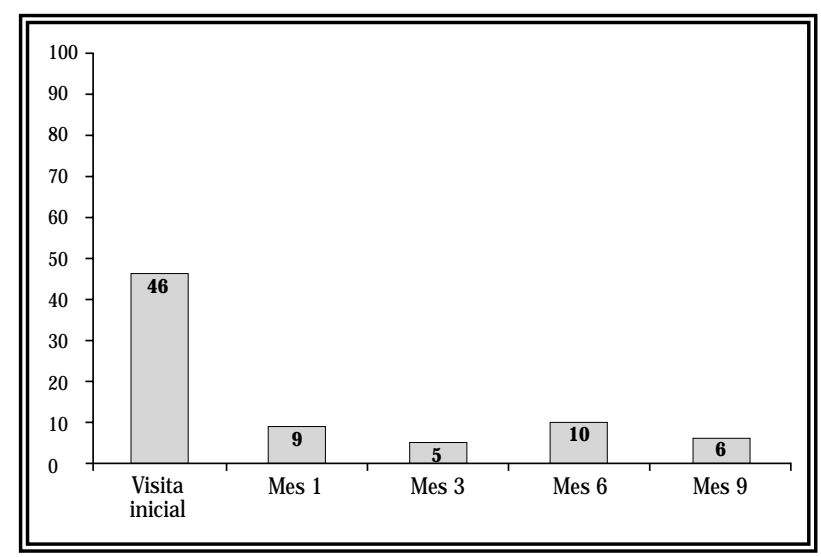

FIGURA 2. Proporción de sintomatología miccional irritativa (\%). 
tivo (Fig. 2), y un 52,6\% tenían infección urinaria. De éstas, el $77,8 \%$ fueron por E. Coli y el $22,2 \%$ por Proteus. Para su tratamiento se empleó ciprofloxacino en el $30 \%$ de las pacientes, amoxicilina/clavulánico en el 30\%, cotrimoxazol en el $20 \%$ y fosfomicina en el $20 \%$ restante.

En el último control del noveno mes, el 5,6\% de las pacientes presentaban síndrome miccional irritativo y un $47,4 \%$ tenían infección urinaria, causada en todos los casos por E. Coli.

\section{DISCUSIÓN}

Existen numerosos trabajos previos que demuestran que el AM3 potencia la acción de las células encargadas de los mecanismos de inmunidad celular. Así, por ejemplo, se ha visto que interviene sobre la modulación de la producción de citoquinas de diferentes formas. Actúa sobre la interleuquina 1 (IL-1), aumentando su producción ${ }^{16}$, modulando su producción ${ }^{17}$ e induciendo la expresión de su RNA mensajero (RNAm) ${ }^{18}$. También provoca una inhibición parcial en la producción endógena de TNF- $\alpha /$ Caquectina $^{17}$. Por otro lado, potencia la producción de interleuquina 12 (IL-I2) ${ }^{19,20}$, lo que explica el incremento de respuestas inmunológicas de mediación celular y las infecciones provocadas por patógenos intracelulares como los virus ${ }^{21}$. También aumenta la proliferación linfocitaria $\mathrm{T}$ y la actividad $\mathrm{NK}^{20}$, así como la producción endógena de IFN- $\gamma$. Finalmente, dentro de su acción moduladora de la producción de citoquinas también induce la producción de factores endógenos hematopoyéti$\cos ^{18,22}$ y potencia la secreción temprana de interferón ${ }^{23}$.

Por otro lado, el AM3 interviene también en los mecanismos de respuesta de mediación celular. Así, por ejemplo, incrementa las actividades fagocíticas y microbicidas de los macrófagos frente a diversos gérmenes ${ }^{16,24-27}$, aumenta el número y la actividad quimiotáctica de los monocitos ${ }^{28}$, aumenta la quimiotaxis y fagocitosis de los polimorfonucleares neutrófilos (PMN), incrementa la actividad citotóxica de los macrófagos ${ }^{28,29}$, aumenta el número y la actividad de las células $\mathrm{NK}^{17,28,30,31}$, promueve los fenómenos de distribución celular tisular (DCT), potencia la respuesta proliferativa de linfocitos $\mathrm{T}^{20}$ e incrementan las cifras de linfocitos T CD4 y CD8.
La actividad del AM3 no sólo se ha ensayado in vitro sino también in vivo con animales y con humanos. En estudios preclínicos con animales se ha observado que retarda el desarrollo de $P$. Carinii en ratas infectadas que habían sido inmunodeprimidas con cortisona ${ }^{32}$. Asociada a penicilina G sódica disminuye la tasa de mortalidad en infección letal por S. Aureus en ratones inmunodeprimidos con cortisona ${ }^{33}$, incrementa las dosis de gérmenes necesarios para matar al 50\% de ratones infectados por E. $\mathrm{Coli}^{27}$, y demuestra efectos antivirales aditivos con la ribavarina ${ }^{17}$.

En seres humanos, un meta-análisis ${ }^{38,47}$ demostró que disminuye significativamente el número de episodios infecciosos agudos recidivantes del tracto respiratorio, su duración, la necesidad de tratamiento antibiótico y el número de bajas laborales en pacientes de edad mediaavanzada afectos de enfermedad pulmonar obstructiva crónica (EPOC). También disminuye el número y duración de las úlceras en la estomatitis aftosa recidivante ${ }^{30}$. En el tratamiento de la tuberculosis pulmonar disminuye el tiempo de negativización del esputo, acelera la cura de lesiones y mejora los síntomas clínicos ${ }^{34}$. Asimismo, el AM3 se ha demostrado un eficaz coadyuvante en la vacunación frente a la hepatitis $B$ ya que revierte el estado de no respondedor a la vacunación ${ }^{35}$, potencia la respuesta a la primovacunación en sanos $^{35} \mathrm{y}$ en hemodializados.

En resumen, el tratamiento con AM3 potencia los mecanismos de la inmunidad natural (células NK y macrófagos). Esto podría explicar el aumento en la resistencia del huésped a la colonización bacteriana. Por otro lado, su acción es diferente a las de las vacunas polisacárido/proteínicas ${ }^{36}$, ya que al contrario que en el AM3, en la acción de estas vacunas no participan los mecanismos de inmunidad natural. Por ello, el AM3 es capaz de promover una respuesta moderada específica de mediación celular que acaba produciendo linfocitos T citotóxicos.

Como ya se ha comentado la CRM, requiere de tratamientos antibióticos profilácticos de larga duración, teniendo un porcentaje importante de recaídas cuando se abandonan ${ }^{3-7}$. Por este motivo, desde hace un tiempo se están ensayando nuevos tratamientos alternativos al tratamiento antibiótico. Dado que el AM3 ha demostrado in vitro e 
in vivo, capacidad para potenciar los mecanismos inmunitarios y mejorar la evolución de otras enfermedades infecciosas ${ }^{30,34,35}$, pensamos que podía tener interés evaluar su acción en la CRM. Con este objetivo debe realizarse un ensayo clínico. Pero previamente es necesario un estudio piloto que evalúe la acción beneficiosa o no del AM3 en las pacientes con CRM y aporte información sobre la proporción de respuesta al tratamiento, para poder calcular posteriormente el tamaño muestral del ensayo clínico. Para ello, se ha efectuado el presente trabajo donde se ha observado una respuesta positiva tanto a nivel de infección como de sintomatología. En él se ha mostrado que la proporción de infección disminuyó del 100\% al 26\% en el primer mes, para aumentar luego y quedar en unas cifras en torno al $50 \%$ a partir del tercer mes (Fig. 1). Esta reducción del 50\% es prometedora en una patología con una recidiva tan elevada. Además, estas pacientes recibieron antibiótico en dosis inferiores a los tratamientos clásicos, ya que sólo se trataron las infecciones cuando estaban presentes en cada una de las visitas de control. En ningún caso se indicaron pautas de antibiótico profiláctico a largo plazo. Esta mejora de la infección usando dosis totales bajas de antibiótico se podría explicar por el aumento en la resistencia del huésped a la colonización bacteriana promovida por la acción del AM3. Por otro lado, se constata también una mejora en la sintomatología miccional irritativa de la paciente, ya que de estar presente en la mitad de las pacientes en la visita inicial, disminuye por debajo del 10\% en los sucesivos controles (Fig. 2).

Todos estos resultados esperanzadores de este estudio inicial han de ser asumidos con cautela hasta que no se confirmen mediante la realización de un ensayo clínico. Hay dos hechos que sugieren la necesidad de realizar un ensayo controlado para confirmar el efecto de AM3 en la CRM. Primero, los estudios previos in vitro e in vivo que demuestran la acción del AM3 sobre los mecanismos inmunitarios y, concretamente, en patologías infecciosas. Segundo, los resultados de este estudio piloto que muestran que AM3 redujo en un 50\% la presencia de infección urinaria manifiesta y en un $90 \%$ la sintomatología miccional irritativa. Si este ensayo clínico acaba confirmando estos resultados preliminares, estaremos ante un avance importante en el tratamiento de esta patología. Fundamentalmente, porque si con el uso coadyuvante de AM3 no se requieren tandas largas de tratamiento antibiótico, ni tratamiento profiláctico supresivo ni profilaxis post-coital, se conseguiría una disminución de la recurrencia, un manejo más sencillo de la enfermedad por parte de la paciente, un ahorro en el gasto farmacéutico provocado por el consumo de dichos antibióticos y una disminución de los efectos secundarios derivados de las pautas largas de tratamiento antibiótico.

\section{REFERENCIAS}

1. FOXMAN B, BARLOW R, D’ARCY H et al.: Urinary tract infection: self-reported incidence and associated costs. Ann Epidemiol 2000; 10: 509-515.

2. SANFORD JP.: Urinary tract symptoms and infection. Ann Rev Med 1975; 26: 485-498.

3. MELEKOS MD, ASBACH HW, GERHARZ E et al.: Post-intercourse versus daily ciprofloxacin prophylaxis for recurrent urinary tract infections in premenopausal women. J Urol 1997; 157: 935-939.

4. KROMERY S, HROMEC J, TVRDIKOVA M et al.: Newer quinolones in the long term prophylaxis or recurrent urinary tract infections. Drugs 1999; 58: 99-102.

5. STAPLETON A, LATHAM RH, JOHNSON C et al.: Postcoital antimicrobial prophylaxis for recurrent urinary infection. A randomized double-blind, placebo-controlled trial. JAMA 1990; 264: 703-706.

6. VOSTI KL.: Recurrent urinary tract infections. Prevention by prophylactic antibiotics after sexual intercourse. JAMA 1975; 231: 934-940.

7. NABER KG, BERGMAN B, BISHOP MC et al.: Guidelines on urinary and male genital tract infections. European Association of Urology: 13-15.

8. NICOLLE LE, RONALD AR.: Recurrent urinary tract infection in adult women: diagnosis and treatment. Infect Dis Clin North Am 1987; 1: 793-806.

9. REID G, BRUCE AW, TAYLOR M.: Influence of threeday antimicrobial therapy and lactobacillus vaginal suppositories on recurrence of urinary tract infections. Clin Ther 1992; 14: 11-16.

10. BAERHEIM A, LARSEN E, DIAGRANES A.: Vaginal application of lactobacilli in the prophylaxis of recurrent lower urinary tract infection in women. Scand $J$ Prim Health Care 1994; 12: 239-243.

11. TAMMEN H.: Immunobiotherapy with Uro-Vaxom in recurrent urinary tract. The german urinary tract infection study group. Br J Urol 1990; 65: 6-9.

12. REID G, BRUCE AW, TAYLOR M.: Instillation of Lactobacillus and stimulation of indigenous organisms to prevent recurrence of UTI. Microecol Ther 1995; 23: 32-45.

13. KONTIOKARI T, SUNDQVIST K, NUUTINEN M et al.: Randomised trial of cranberry-lingonberry juice and Lactobacillus GG drink for the prevention of urinary tract infections in women. BMJ 2001; 322: 15711577. 
14. AVORN J, MONANE M, GURWITZ JH et al.: Reduction of bacteriuria and pyuria after ingestion of cranberry juice. JAMA 1994; 271: 751-754.

15. HOWELL A, VORSA N, DER MARDEROSIAN A et al.: Inhibition of the adherance of P-fimbriated Escherichia coli to uroepithelial-cell surfaces by proanthocyanidin extracts from cranberries. $N$ Engl J Med 1998; 339: 1085-1086.

16. BARASOAIN I, REJAS MT, ARAMBARU J.: Inmunoferon ${ }^{\circledR}$ enhances IL-1 production and restores the phagocytic activity of macrophages in immunodepressed animals. Proc XII Cong Nal Microbiol 1989: Abstract 37.

17. VILLARRUBIA VG, HERRERÍAS JM, ÁLVAREZ-MON $M$ et al.: Virus de la hepatitis B y la respuesta inflamatoria/inmunológica II. Una nueva oportunidad de tratamiento de la infección crónica y una sugerencia para el tratamiento de otras enfermedades viricas persistentes. Rev Esp Enf Digest 1998; 90: 514-522.

18. GUENECHEA G, BUEREN JA, MAGANTO G et al: AM5, a protein-associated polysaccharide, stimulates hematopoiesis and modulates the expression of endogenous hematopoietic growth factors in murine long-term bone marrow cultures. Stem Cells 1995; 13: 175-184.

19. SKEEN MJ, MILLER MA, SHINNICK TM et al.: Regulation of murine macrophage IL-I2 production. Activation of macrophages in vivo, restimulation in vitro, and modulation by other cytokines. J Immunol 1996; 156: 1196-1206.

20. ROJO JM, REJAS MT, OJEDA G et al.: Enhancement of lymphocyte proliferation, interleukin-2 production and NK activity by Inmunoferon ${ }^{\circledR}$, a fungal immunomodulator: variations in normal and immunosuppressed mice. Int J Immunopharmacol 1986; 8: 593597.

21. ORANGE JS, WANG B, TERHORST C et al.: Requirement for natural killer cell produced interferon gamma in defense against murine cytomegalovirus infection and enhancement of this defense pathway by interleukin I 2 administration. J Exp Med 1995; 182: 1045-1056.

22. REAL A, GUENECHEA G, BUEREN JA et al.: Radioprotection mediated by the haemopoietic stimulation conferred by AM5: a protein-associated polysaccharide. Int $J$ Radiat Biol 1992; 62: 65-72.

23. MOYA P, BAISERAS P, BARASOAÍN I et al.: Inmunoferon ${ }^{\circledR}$ enhances the activities of early-type interferon inducers and natural killer cells. Immunopharmacol Immunotoxicol 1987; 9: 243-246.

24. GILLISSEN G, BREUER-WERLE M.: Influencia de un inmunomodulador sobre la fagocitosis en el test de clearance. Rev Clin Esp 1984; 1: 17-19.

25. CAÑAVATE ML, PONTÓN J, AMURRIO C et al.: Efecto de un nuevo inmunomodulador sobre la funcionalidad de macrófagos de ratón. Rev Clin Esp 1984; 3: 159-162.
26. LARRAD L, BENITO R, LASIERRA MP.: Efecto de AM3 (Inmunoferon $\AA$ ) sobre la capacidad microbicida de macrófagos esplénicos de conejo en la infección provocada por Brucella abortus. Infectológika 1985; 1: 38-40.

27. RODRÍGUEZ F, BRIEVA A, TUDURI $P$ et al.: Estudio de la influencia de un nuevo fármaco sobre el sistema inmune y su relación con la resistencia a la infección provocada en ratón. Rev Clin Esp 1983; 169: 191-193.

28. VILLARRUBIA VG, MORENO KOCH MC, CALVO C et al.: The immunosenescent phenotype in mice and humans can be defined by alterations in the natural immunity. Reversal by immunomodulation with oral Inmunoferon ${ }^{\circledR}$. Immunopharmac \& Immunotox 1997; 19: 53-74.

29. VILLARRUBIA VG, SADA G, SOZZANI S et al.: AM3 (Inmunoferon ${ }^{\circledR}$ ) restaura las actividades de macrófagos y de células NK en ratones inmunocomprometidos. Oncología 1987; 10: 283-291.

30. CAMACHO F, ELORZA FL, ORTEGA Y et al.: Inmunoferon ${ }^{\circledR}$ (un modificador de la respuesta biológica por vía oral) en el tratamiento de la estomatitis aftosa recidivante (EAR). Evaluación clínica y estudios preliminares sobre células NK (CD16) y su papel patogénico en la EAR. Rev Clin Esp 1991; 188: 403-408.

31. VILLARRUBIA VG, VALLADOLID JM, ELORZA FL et al.: Therapeutic response of chronic active hepatitis $B$ (CAHB) to a new immunomodulator: Inmunoferon ${ }^{\circledR}$. Immunohematological effects. Immunopharmac Immunotox 1992; 14: 141-164.

32. PAINE R, PRESTON AM, WILCOXEN S et al.: Granulocyte-macrophage colony-stimulating factor in the innate immune response to Pneumocystis carinii pneumonia in mice. $J$ Immunol 2000; 164: 2602-2609.

33. GILLISSEN G, BREUER-WERLE M.: L'action additive d'un inmunomodulateur sur l'effect thérapeutique des antibiotiques. Path Biol 1983; 31 (6): 548-550.

34. CAMEROTA G, ZUBIANI G, DI PROIETTO MC.: Empleo de un fármaco inmunoestimulante en la terapia de la tuberculosis pulmonar. Infectológika 1984; 5: 294-299.

35. GARCÍA CALVO C.: AM3 (Inmunoferon®) como adyuvante a la vacunación contra la hepatitis B. Tesis Doctoral. Facultad de Medicina, Universidad Complutense de Madrid, 1996.

36. ROBBINS JB, SCHNEERSON R.: Polysaccharideprotein conjugates: a new generation of vaccines. $J$ Infect Dis 1990; 264: 503-505.

Dr. F. Millán

Servicio de Urología. Fundació Puigvert

C/ Cartagena, 340-350

08025 Barcelona

(Trabajo recibido el 24 junio de 2002) 